\title{
Saillant, Francine, Kilani, Mondher \& Graezer Bideau Florence (dir.) - Manifeste de Lausanne
}

Stéphanie Pouessel

\section{(2) OpenEdition}

1 Journals

Édition électronique

URL : http://journals.openedition.org/etudesafricaines/14534

DOI : 10.4000/etudesafricaines. 14534

ISSN : 1777-5353

Éditeur

Éditions de l'EHESS

\section{Édition imprimée}

Date de publication : 16 septembre 2013

Pagination : 764-767

ISBN : 978-2-7132-2388-4

ISSN : 0008-0055

Référence électronique

Stéphanie Pouessel, « Saillant, Francine, Kilani, Mondher \& Graezer Bideau Florence (dir.) - Manifeste de Lausanne », Cahiers d'études africaines [En ligne], 211 | 2013, mis en ligne le 20 septembre 2013, consulté le 24 septembre 2020. URL : http://journals.openedition.org/etudesafricaines/14534 ; DOI : https://doi.org/10.4000/etudesafricaines.14534

Ce document a été généré automatiquement le 24 septembre 2020.

(c) Cahiers d'Études africaines 


\title{
Saillant, Francine, Kilani, Mondher \& Graezer Bideau Florence (dir.) - Manifeste de Lausanne
}

\author{
Stéphanie Pouessel
}

\section{RÉFÉRENCE}

SAILLANT, Francine, KILANI, Mondher \& GRAEZER BIDEAU Florence (dir.) - Manifeste de Lausanne. Pour une anthropologie non hégémonique. Montréal, Liber, 2011, 143 p., bibl.

1 Si l'on jugeait la tradition intellectuelle du «Nord» incompatible avec la posture postcoloniale, c'est maintenant chose révolue. Sous forme de "manifeste», ce petit ouvrage s'empare de la vision altermondialiste ambiante pour l'appliquer à l'anthropologie. Il entend éviter la reproduction, dans le travail anthropologique, des inégalités qui marquent les marchés économiques et politiques et compare ainsi l'« anthropologie non hégémonique » qu il défend au sommet des " pays non alignés » (1955), à l'anti-interventionnisme en Irak (2003) ou à l'anti-sommet de Davos.

2 Ce manifeste est le fruit de plusieurs rencontres d'un groupe d'anthropologues depuis celle de Québec en 2007 (trentième anniversaire de la revue Anthropologie et sociétés) jusqu'à celle de Libreville en 2011, mais qui a pris «Lausanne» comme ancrage géographique pour relever sa neutralité. Dirigé par Francine Saillant, Mondher Kilani et Florence Graezer Bideau, un texte introductif ( 24 pages) et des entrées thématiques composent l'ouvrage auquel onze contributeurs ont pris part.

3 Face à la racialisation des rapports de force du social et à l'investissement du langage des sciences sociales par des groupes jusque-là étudiés, l'anthropologie se sent ici prise à partie et décontenancée. l'ouvrage part en effet du constat inquiet de la fin de ce que furent «les anciens objets d'étude" autrement dit les groupes ou sociétés traditionnelles. Ces derniers sont désormais largement intégrés dans la globalisation du savoir. ils sont conscients des études les concernant et au fait de l'intérêt en terme de 
droit ou de reconnaissance que cela peut constituer pour eux. Pourtant, diverses solutions ont déjà été proposées face à cette "révolution du lectorat $»^{1}$ et l'ouvrage aurait gagné à s'y référer : une « anthropologie collaborative » qui, selon Marie Miran, "valorise (sans se résumer) la coproduction et la co-signature de textes avec des acteurs non professionnels issus des communautés étudiées $»^{2}$. Avant elle, Johannes Fabian proposait de désanonymer les personnes à la base de nos analyses, voire de les répertorier en tant que co-auteurs ${ }^{3}$.

Selon les auteurs de ce Manifeste, ce nouveau contexte bouleverse la donne des rapports Nord-Sud et des "anthropologies occidentales et celles non occidentales". Dans notre époque « de la pensée unique et des intégrismes » (p. 20), le collectif du livre s'inscrit dans la perspective de l'expression «anthropologies hégémoniques » de Restrepo et Escobar ${ }^{4}$ qui veut rendre compte des pratiques et discours institutionnels producteurs des normes, des standards et des canons de la discipline établis par les départements universitaires du « Nord » (États-unis, France, Grande-Bretagne).

À travers la remise en cause de "l'unidirectionnalité du rapport entre sujet et objet » (p.56), il vise à la "décolonisation de l'anthropologie » (p.57), une anthropologie décentrée et engagée pour contribuer à "une nouvelle forme de justice cognitive " (p. 117) envers des « savoirs hégémoniques qui justifient les inégalités fondées sur des différences de sexe, de race, de classe et d'âge » (p. 118). À travers cette « anthropologie non autoritaire» (p.15), "relationnelle et dialogique multilocalisée quant aux lieux d'énonciation des particularismes culturels et de leurs savoirs» (p. 24), les auteurs invitent à penser l'engagement et l'éthique au sein de la discipline. Pourtant, cette réflexion réflexive est-elle vraiment inédite? Ces deux "valeurs » (engagement et éthique) sont-elles exclusivement le souci d'une science sociale du « Nord», comme le postule l'ouvrage? Ne sommes-nous pas tous confrontés à ces marges éthiques et humaines constitutives de l'ethnographie?

6 Cette critique de la référence universaliste classique, au fondement d'un discours anthropologique jugé nécessairement hégémonique appelle à relativiser la connaissance venant du Nord : «De toute manière, avec ou sans nous, les départements d'anthropologie de l'hémisphère sud feront tôt ou tard contrepoids à ceux de l'hémisphère nord qui étaient jusqu ici les seuls à prospérer et à imposer leurs théories » (p. 94). La distinction Nord-Sud que le propos du livre réitère, en prétendant (pourtant) la combattre, est aujourd'hui caduque, tant de la part des sciences sociales que des mouvements sociaux eux-mêmes. Le Maghreb en est un cas exemplaire : des sociétés qui ne rentrent pas dans les cases exotiques de l'ethnologie (étant donné la proximité de la langue française et des «Franco-Maghrébins») et qui furent, via l'étincelle tunisienne, initiatrices d'un mouvement de protestations mondial en 2011 (les «indignés »). Autant d'éléments qui brisent définitivement la vision orientaliste, soumise et provinciale de ces sociétés.

Qu'est-ce que signifie «être du Nord»? Y être né ? Avoir grandi sur ces terres? Descendre de géniteurs du Nord (ce qui ne fait que repousser la question) ? Y vivre depuis dix ans? Quinze ans ? Y avoir été formé ? À partir du baccalauréat? Du master? Ces déclinaisons de parcours de vie, dont les critères sont multipliables à l'infini, illustrent la relativité du concept de Nord aujourd'hui qui, réifié, crée en miroir une fictive " communauté décolonisée » (Achille Mbembe) ${ }^{5}$. Le parcours de Mondher Kilani, co-auteur du livre, est pourtant l'expression même d'un ethnologue contemporain inclassable selon l'ancienne dichotomie Sud-Nord: son baccalauréat passé à Tunis, son 
doctorat à Paris, il choisit l'altérité radicale et insulaire comme premier terrain ethnographique (Papouasie-Nouvelle-Guinée) puis optera pour les Alpes suisses, les oasis du Sud-tunisien, le Niger, la Malaisie.

8 En renouvelant les poncifs de l'ethnologie - la nécessité absolue d'un terrain long, l'apprentissage des langues locales, etc. - , le propos du livre semble déconnecté des avancées de la discipline (ethnographie multisite, étude en réseaux, co-écriture, etc.). Il fait l'éloge de l'«engagement» sous couvert d'«expériences humaines", sans en préciser sa forme: conversion religieuse? Mariage avec un partenaire du groupe étudié? Simple présence? D'autre part, l'apprentissage d'apparence évident de la "langue locale » est lui-même à questionner. Aujourd'hui, au sein de sociétés très souvent plurilingues, un étranger (c'est-à-dire l'ethnologue, quoi qu'il en pense) usant de la langue vernaculaire peut faire figure d'injure sous-entendant leur non-maîtrise de la langue véhi-culaire. Encore une fois, le cas du Maghreb est à ce titre évocateur : dans le domaine de la diplomatie, la nomination d'un ambassadeur français arabisant en Tunisie (et de plus qui maîtrise l'arabe littéral et non l'arabe dialectal) est apparu pour certains comme un affront qui niait la francophonie du pays, c'est-à-dire, aussi, selon certains Tunisiens, sa modernité (janvier 2011).

9 Envers une langue anglo-saxonne clairement catégorisée d'hégémonique, le Manifeste fait l'éloge du «multilinguisme » que devrait emprunter l'écriture de l'anthropologie : français, espagnol, portugais, arabe sont encouragés en tant que langues non hégémoniques. l'appréhension des langues est pourtant relative: une même langue peut être perçue comme subalterne pour certains et impériale pour d'autres. Quand la langue arabe est perçue depuis l'Occident comme une langue-stigmate, celle des anciens colonisés, des banlieusards, voire d'une Palestine bafouée, elle représente dans les milieux militants amazighs (berbères) la langue coloniale par excellence.

Ce Manifeste entretient le malentendu entre rapport de force imposé par la recherche et rapport de force inhérent à la société, à l'instar des processus de différenciation qui sont tout bonnement appelés à être relativisés; le livre assimile l'autoritarisme de l'anthropologie (c est pourtant les sciences sociales qu'il aurait fallu viser) qu il s'agit évidemment d'enrayer, mouvement amorcé depuis déjà presque un siècle, et les processus hégémoniques du monde social en lui-même qui n'ont que faire des théories humanistes des ethnologues. Si l'anthropologie permet, à long terme et de manière indirecte, d'atténuer les processus de dominations, cela ne peut constituer le fil conducteur d'une recherche, au risque d'en être aveuglé.

11 Ce Manifeste de Lausanne, qui emprunte l'air du temps pour questionner la démarche même de l'anthropologie, espère briser l'antique distinction entre « Nous » et « Eux ». Il est à espérer que les nouvelles générations d'anthropologues ne se reconnaissent déjà plus dans ces catégories d'origine coloniale. 


\section{NOTES}

1. C. B. BRetTell (ed.), When they Read what We Write: the Politics of Ethnography, Westport, Bergin and Garvey, 1993.

2. M. MIRAN, «Quand ethnologue et imam croisent leurs plumes. Récit d'un voyage au pays de l'anthropologie collaborative », Cahiers d'Études africaines, 198-199-200, 2010, p. 954.

3. F. JOHANNES, Ethnography as Commentary, Writing from the Virtual Archive, Durham-London, Duke University Press, 2008.

4. A. Escobar \& E. ReSPETRo, "Anthropologies hégémoniques et colonialité », Cahiers des Amériques latines, 62, 2009, pp. 83-95.

5. A. MBEMBE, Sortir de la grande nuit. Essai sur l'Afrique décolonisée, Paris, La Découverte, 2010, p. 16. 